\title{
UWB pulse detection and TOA estimation using GLRT
}

\author{
Yan Xie $^{1^{*}}$ (D), Gerard J.M. Janssen ${ }^{1}$, Siavash Shakeri² and Christiaan C.J.M. Tiberius ${ }^{2}$
}

\begin{abstract}
In this paper, a novel statistical approach is presented for time-of-arrival (TOA) estimation based on first path (FP) pulse detection using a sub-Nyquist sampling ultra-wide band (UWB) receiver. The TOA measurement accuracy, which cannot be improved by averaging of the received signal, can be enhanced by the statistical processing of a number of TOA measurements. The TOA statistics are modeled and analyzed for a UWB receiver using threshold crossing detection of a pulse signal with noise. The detection and estimation scheme based on the Generalized Likelihood Ratio Test (GLRT) detector, which captures the full statistical information of the measurement data, is shown to achieve accurate TOA estimation and allows for a trade-off between the threshold level, the noise level, the amplitude and the arrival time of the first path pulse, and the accuracy of the obtained final TOA.
\end{abstract}

Keywords: Ultra-wide band (UWB), Time-of-arrival (TOA) estimation, Sub-Nyquist sampling, GLRT, Ranging, Positioning

\section{Introduction}

The demand for accurate wireless positioning in indoor environments is vastly increasing for precise localization and tracking of objects and people, with applications in a.o. industry, warehouses, shopping malls, and hospitals. In these environments, traditional positioning solutions based on satellite signals are unreliable, unavailable, or do not provide sufficient accuracy. In recent years, much attention has been given to ultra-wideband (UWB) signals for indoor positioning. UWB signals, e.g., sub-nanosecond duration pulse signals, enable precise ranging because they allow for separation of multipath components and accurate estimation of the time-of-arrival (TOA) of the first arriving path (FP) signal $[1,2]$. With this feature of UWB signals, centimeter-level ranging accuracy can be achieved, even in dense multipath environments [2,3].

In recent decades, researchers have been developing several approaches to get the TOA of the received FP pulse. In [3, 4], pulses with sub-nanosecond duration are transmitted and then measured with a digital sampling scope with full sampling rate as the receiver. Based on the recovered received signal, time-domain TOA

\footnotetext{
*Correspondence: y.xie@tudelft.nl

${ }^{1}$ Faculty of Electrical Engineering, Mathematics and Computer Science, Delft University of Technology, Mekelweg 4, 2628 CD Delft, The Netherlands

Full list of author information is available at the end of the article
}

estimation algorithms, which are typically maximum likelihood (ML)-based approaches [5-7], are applied by identifying the FP from the sampled signal. Besides, [8-13] get the received pulses by setting up a frequency domain measurement system using a vector network analyzer (VNA) to record the frequency response of the channel. This measured frequency response can be converted to the time domain by taking the inversed Fourier transform, or a variety of super-resolution techniques, such as root multiple signal classification (MUSIC) [14] and total least square-estimation of signal parameter via rotational invariance (TLS-ESPRIT) [15], can be applied to increase time-domain resolution based on frequency response measurements. On the other side, the wide bandwidth of UWB signals, usually in the gigahertz $(\mathrm{GHz})$ range, puts high demands on the sampling circuit to satisfy Nyquist sampling rates, which are easily several Giga-samples per second $(\mathrm{GS} / \mathrm{s})$. In order to overcome this disadvantage, researchers resort to sub-Nyquist sampling techniques for ranging using sub-nanosecond pulse signals. In [16, 17], compressed sampling techniques are applied to realize sub-Nyquist sampling at the expense of a more complex receiver structure. Fontana and Gunderson [18] gives a novel design to obtain TOA measurements directly without sampling of the received signal, where a receiver is used based on a daisy-chain structured tunnel diode

\section{Springer Open}

(c) The Author(s). 2017 Open Access This article is distributed under the terms of the Creative Commons Attribution 4.0 International License (http://creativecommons.org/licenses/by/4.0/), which permits unrestricted use, distribution, and reproduction in any medium, provided you give appropriate credit to the original author(s) and the source, provide a link to the Creative Commons license, and indicate if changes were made. 
detector to detect successive peaks of the measured channel impulse response. The benefit of this method is that, TOA measurements are obtained directly without the use of high-sampling rate circuits or universal test instruments, which allows the receiver to be highly integrated, of low complexity and low cost. However, compared with Nyquist sampling-based and compressive sampling-based TOA estimation schemes, TOA estimation schemes based on direct TOA measurements have not been investigated in-depth in the literature.

In the following, we further investigate the idea of direct TOA measurements, where the time instant at which the received signal crosses a preset threshold voltage is detected using a sub-Nyquist sampling technique. This threshold voltage is chosen such that the probability of threshold level crossing is due to noise only and the probability of false alarm, $P_{F A}$, is at an acceptable low level. Therefore, the first threshold-exceeding signal in each measurement period of the receiver can be considered caused by the transmitted pulse signal traveling via the shortest path, and the measured TOA of this signal is recorded. However, a measurement event might be wrongly recorded as a TOA when the threshold level was crossed due to noise before arrival of the pulse, or the pulse might be miss-detected due to its low power level compared to the threshold and a late path signal may be detected. Thus, the threshold value, which should be set based on both the noise level and the expected pulse level, determines the probability of correct pulse detection as well as the sensitivity of the receiver.

The analysis of threshold selection has not been addressed in detail in early researches. In [1], an approach is given on the kurtosis of the signal samples for threshold selection by applying an energy detector to capture both the statistics of the channel and the SNR of the received signal. However, this TOA estimation method is based on full Nyquist sampling of the received signal and TOA estimation accuracy is at meter level.

Receiver and detection techniques which operate at a much lower sample rate than Nyquist were proposed in $[2,19]$ and $[20]$. In this detector, the crossing of a preset threshold level triggers the discharging of an $\mathrm{RC}$ circuit with an accurately known RC-time and therefore discharge curve. With only a few samples of this curve, the precise start of the discharging process can be estimated. Triggering of the discharging process can happen due to a received signal pulse but also (occasionally) due to noise. However, noise may also cause a deviation of the trigger moment from the actual arrival instant of the pulse. Since it is not feasible to apply signal averaging to improve the probability of detection $\left(P_{D}\right)$ of this sub-Nyquist-based receiver structure, we need to resort to another approaches to maximize $P_{D}$. In [2] and [3], TOA estimation performance is statistically analyzed for the threshold level selected based on the pre-set $P_{F A}$. The technique for estimating the TOA proposed in [2] is based on finding a sliding time-window which contains at least a certain predetermined minimum number of TOA events, which depends on channel quality (SNR, SIR) and the number of measured events. The mean TOA of the events within the sliding window was selected as the estimated TOA. A disadvantage of this technique is that a large time window following the arming of the detector had to be excluded from TOA estimation because of the high number of level crossings due to noise only in that range, and therefore making the detector less efficient. The results in [2] show that a higher estimation accuracy can be achieved compared to energy-based detection schemes; however, since the distribution of the measured TOA events due to noise and the preset detector threshold is not exploited, there is room for improvement of both the TOA estimation accuracy and the receiver's sensitivity.

In this paper, an improved TOA estimation method is proposed which takes into account the distribution of the measured TOA events: the time distribution of the measured first level-crossing events due to noise only, which strongly depends on the time-duration since arming the detector, and the distribution of the first level-crossing events in case a pulse plus noise was received. The pulse detection and TOA estimation are based on the GLRT detector, using the likelihood ratio of the TOA distribution. By exploiting the full statistical information of the measurement data, receiver sensitivity and the accuracy of the estimated TOA can be substantially improved.

This paper is organized as follows. In Section 2, the signal model and the low-complexity TOA sub-Nyquist sampling receiver structure are described. In Section 3, a mathematical model of the first threshold crossing is derived for this receiver which is subsequently used to obtain the probability density function (PDF) of the measured TOA events. The noise variance as well as the FP pulse amplitude can be estimated based on this PDF and the recorded statistics of the measured TOA events. Moreover, the relation among threshold-level, noise variance, and the FP pulse amplitude is analyzed. In Section 4, the TOA is estimated using composite hypothesis testing based on the joint PDFs of the TOA measurements. Simulations show that the GLRT results in a vast improvement of the TOA estimation performance. Conclusions are drawn in Section 5.

\section{System model}

\subsection{Signal model}

The transmitted signal is composed of a sequence of pulses, which can be written as a function of time $t$ :

$$
s(t)=\sum_{k=-\infty}^{\infty} w\left(t-k T_{f}\right),
$$


where $w(t)$ is the waveform of a single pulse, $T_{f}$ is the repetition period of the transmitted pulses, and $k$ is the index of a specific time frame.

Accordingly, the received signal in a static multipath channel can be represented as:

$$
r(t)=\sum_{k=-\infty}^{\infty} \sum_{l=0}^{L-1} h_{l} w\left(t-t_{l}-k T_{f}\right)+n(t),
$$

where $h_{l}$ and $t_{l}$ are the amplitude and delay of the $l$-th multipath component, respectively, and $L$ is the number of multipath components, and $n(t)$ is zero-mean additive white Gaussian noise with power spectral density (PSD) $\sigma_{0}^{2}$. For the purpose of ranging, the means for multiple access are not considered in this signal model.

\subsection{Receiver model}

The analysis in this paper is based on a low-complexity receiver structure for UWB TOA measurements, which is a slightly adapted version of the receiver proposed in $[2,19,20]$. Instead of applying full Nyquist sampling on the received signal, this receiver detects the signal which first crosses a pre-set threshold voltage in each measurement period and records the arrival time of this signal, which is assumed to be the FP signal, with respect to the start of the measurement period. For simplicity, the measurement period is taken equal to the pulse repetition period $T_{f}$. Figure 1 shows the block diagram of the proposed receiver.

After amplification and band-pass filtering (BPF) of the signal $r(t)$ received by the antenna, the signal $v(t)$ is compared with a pre-set threshold level $V_{N}$ in a fast comparator. The periodic measurement window is started by the local clock. When $v(t)>V_{N}$ for the first time after the start of a measurement window, the rising edge of the comparator output $V_{C M P}$ latches $V_{S P}$. The latched signal $V_{S P}$ triggers the TOA measurement process and blocks the next threshold crossings, e.g., due to multipath signals, during the remainder of the measurement period. The time-measurement unit determined the TOA of the FP with respect to the start of the current measurement window using the time estimation presented in $[19,20]$. We denote the FP's TOA measurement in the $k$-th repetition period as $t_{M}[k]$ and we collect $K$ TOA measurements in $K$ successive measurement windows.
The input signal $v(t)$ of the comparator in Fig. 1 includes the BPF filtered noise shown in Fig. 2a, and the filtered multi-path pulse signal. To simplify the analysis, we assume that the BPF is an ideal filter with a lower cutoff frequency of $f_{L}$ and an upper cutoff frequency of $f_{H}$, respectively, and the pulses in $v(t)$ to be rectangular with duration $t_{w}$. Figure $2 \mathrm{~b}$ shows the FP pulse with amplitude $A$ ( $A>0$ when it is a positive pulse, or $A<0$ for a negative pulse), rising edge at $t_{r}$, and falling edge at $t_{f}$, where $t_{f}-t_{r}=t_{w}$. In the noise-only case $(A=0)$, the threshold voltage $V_{T H}$ of the system is equal to the pre-set level $V_{T H}=V_{N}$ of the comparator, as shown in Fig. 2c. When the FP pulse is received $(A>0$ or $A<0)$, the input signal $v(t)$, which includes both pulse and noise, is compared to $V_{T H}=V_{N}$, as shown in Fig. 2d. However, this case can also be considered as a special noise-only case where the threshold $V_{T H}$ is changed to $\left(V_{N}-A\right)$ during the time interval $\left[t_{r}, t_{f}\right]$ and stays at $V_{N}$ for the rest of time, as illustrated in Fig. 2e. In this way, pulse detection can be modeled in a unified way as a first-threshold-crossing problem of filtered noise only, with

$$
V_{T H}=\left\{\begin{array}{ll}
V_{N}-A, & t_{r} \leq t_{M}[k] \leq t_{f} \\
V_{N}, & \text { otherwise }
\end{array} .\right.
$$

From a large number of measured pulse arrival times, the probability density function (PDF) of a single pulse measurement and the joint PDFs of all $K$ collected pulse measurements under two different conditions: noise-only case and pulse received case, can be obtained for this firstthreshold-crossing model. These two PDFs can be used in a composite hypothesis test for estimating the FP's TOA. Processing of the TOA measurements $\left.t_{M}[k]\right|_{k=1, \cdots, K}$ to estimate its true TOA is the topic of Section 3.

\section{TOA estimation}

\subsection{First-threshold-crossing problem}

Consider the noise-only case, where, after bandpass filtering as shown in Fig. 1, the voltage $v(t)$ has a PSD of $\mathcal{P}_{v v}(f)=\sigma_{0}^{2}$ for $f_{L}<|f|<f_{H}$ and $\mathcal{P}_{v v}(f)=0$ outside this pass-band. Now, we find the probability of $v(t)<$ $V_{T H}$ in the time interval $(0, t)$, when $v(t)$ first crosses the level $V_{T H}$ in the time interval $(t, t+d t)$. This problem is called the first-crossing problem [21, 22]. The PDF of the

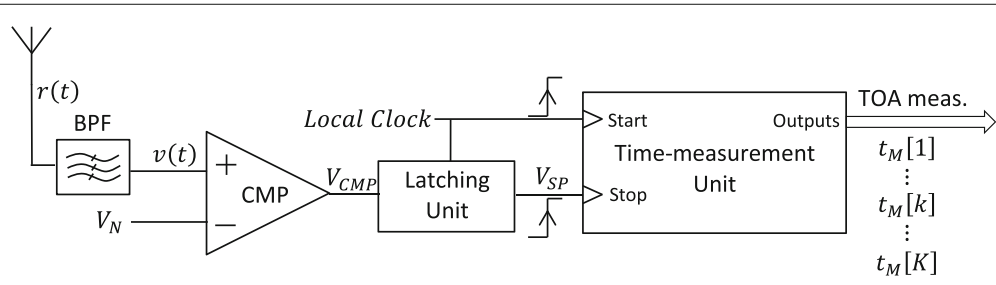

Fig. 1 Block diagram of the proposed receiver 


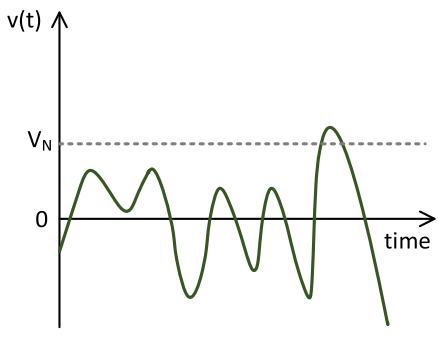

(a) input pass-band noise

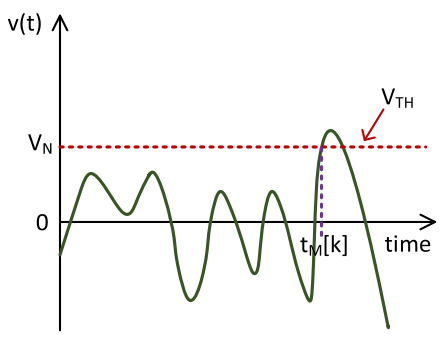

(c) $V_{T H}$ in the noise-only case

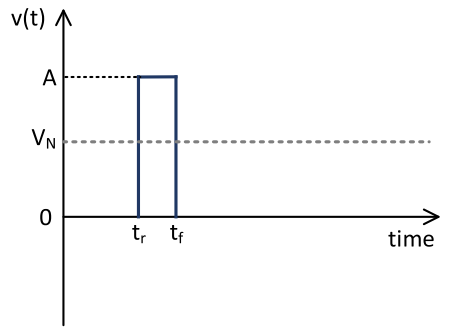

(b) input pass-band FP pulse

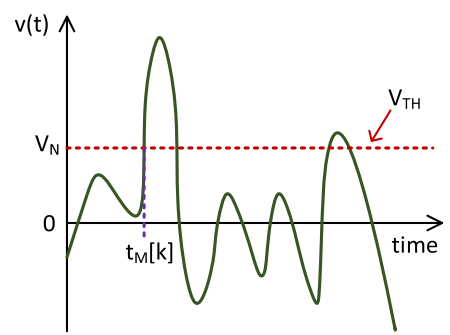

(d) $V_{T H}$ in the FP pulse received case

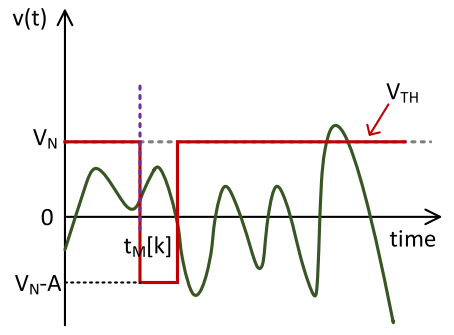

(e) $V_{T H}$ in the FP pulse received equivalent case

Fig. 2 Equivalence of the signal level-crossing model: a the input signal only containing band-pass (BP) noise, $\mathbf{b}$ the input signal only containing an FP pulse, $\mathbf{c}$ a threshold crossing due to BP noise only with $V_{N}=V_{T H}$, d A threshold crossing when the input is the summation of BP noise and an FP pulse with $V_{N}=V_{T H}$ e is the equivalent model of $(\mathbf{d})$, where the input signal contains only BP noise while $V_{T H}$ changes to $V_{N}-A$ during an FP pulse arrival

first-crossing by $v(t)$ at time $t, p_{c}\left(V_{T H}, t\right)$, is an exponential distribution given by

$p_{c}\left(V_{T H}, t\right)=\mu\left[V_{T H}, t \mid(0, t)\right] e^{-\mu\left[V_{T H}, t \mid(0, t)\right] t} \quad(t>0)$,

where $\mu\left[V_{T H}, t \mid(0, t)\right]$ is the probability of an upward crossing in $(t, t+d t)$ with no prior upward crossing in $(0, t),[22]$.

Specifically, considering the statistically rare crossings of a high-level $V_{T H},[21,23,24]$ suggest the following approximation to (4):

$$
p_{c}\left(V_{T H}, t\right) \simeq N_{T H}^{+} e^{-N_{T H}^{+} t} \quad(t>0),
$$

where $N_{T H}^{+}$is the expected number of upward crossings of $v(t)=V_{T H}$ per unit of time.

In the noise-only case, $v(t)$ can be approximated as white noise when the filter bandwidth $B=f_{H}-f_{L}$ is relatively large compared to its center frequency $f_{c}=\frac{f_{H}+f_{L}}{2}$, as shown in Fig. 3a. In this case, $v(t)$ and $\dot{v}(t)=\frac{d v(t)}{d t}$ can be considered as statistically independent, thus $N_{T H}^{+}$can be obtained from

$$
N_{T H}^{+}=\left.\int_{0}^{\infty} \dot{v} p(v, \dot{v} ; t) d \dot{v}\right|_{\nu(t)=V_{T H}},
$$

where $p(v, \dot{v} ; t)$ is the joint PDF for the Gaussiandistributed variables $v(t)$ and $\dot{v}(t)$. Since the crossing duration for a particular positive derivative $\dot{v}$ can be denoted as 


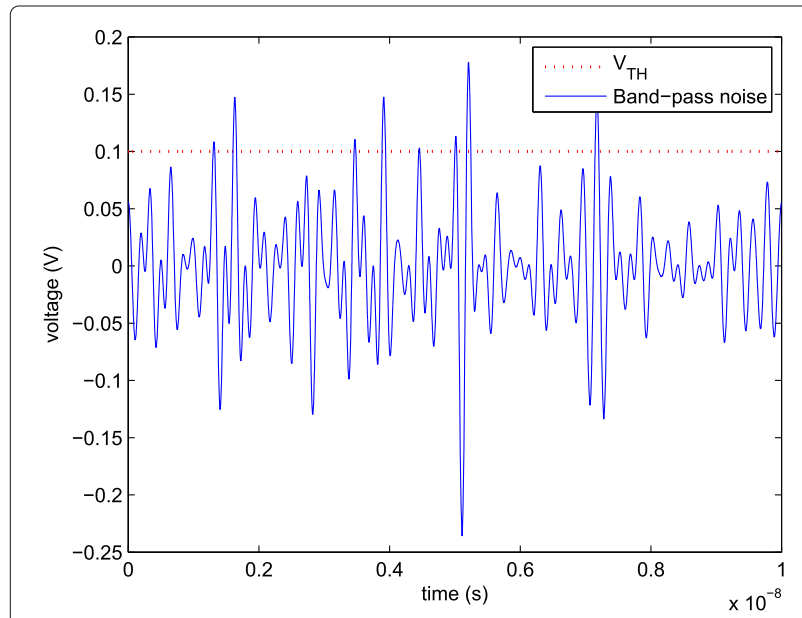

(a) Band-pass noise with $f_{c}=4.6 \mathrm{GHz}$ and $B=5 \mathrm{GHz}$

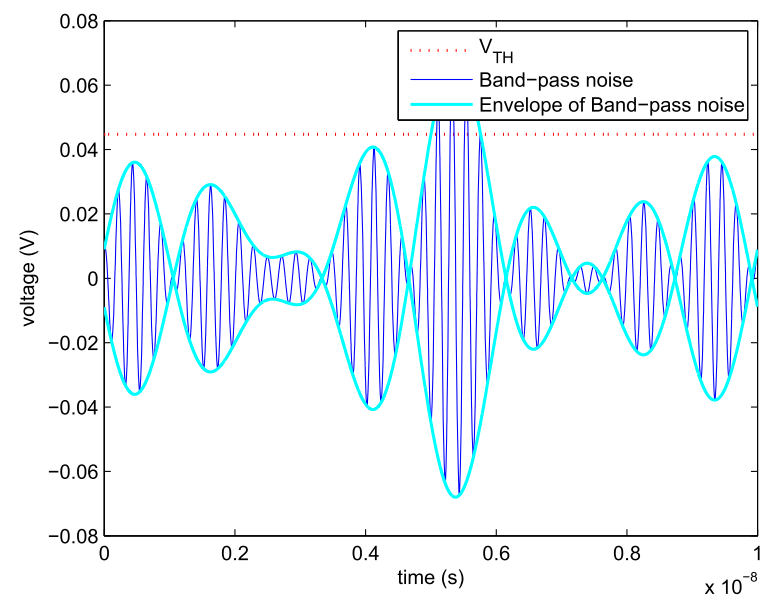

(b) Band-pass noise with $f_{c}=4.6 \mathrm{GHz}$ and $B=1 \mathrm{GHz}$

Fig. 3 Bandpass noise waveforms: $\mathbf{a}$ a waveform of BP noise with relatively large $B / f_{C}$ which corresponds to the case of (17), $\mathbf{b}$ a waveform of $\mathrm{BP}$ noise with relatively small $B / f_{C}$ which matches the case of (18)

$\Delta t_{\dot{v}}=\Delta v / \dot{v},\left.\frac{p(v, \dot{v} ; t) \Delta v \Delta \dot{v}}{\Delta t_{\dot{v}}}\right|_{\nu(t)=V_{T H}}=\left.\dot{v} p(v, \dot{v} ; t) \Delta \dot{v}\right|_{\nu(t)=V_{T H}}$ indicates the expected number of positive passages of $v(t)$ per unit time through the interval $\left[V_{T H}, V_{T H}+\Delta v\right]$ for a given value of $\dot{v}$, where $\Delta v \rightarrow 0$ and $\Delta \dot{v} \rightarrow 0$.

When $B$ is small compared to $f_{c}, v(t)$ has the typical characteristics of a band-pass signal of which the envelope changes are proportional to $B^{-1}$, as shown in Fig. $3 \mathrm{~b}$. Accordingly, $v(t)$ can be expressed as a random envelop modulating a sinewave carrier signal with frequency $f_{c}$ and a random phase angle $\theta(t)$, given by

$$
v(t)=c(t) \cos \left(2 \pi f_{c} t+\theta(t)\right),
$$

where $c(t)$ is the Rayleigh distributed envelope and $\theta(t)$ the uniformly distributed phase of $v(t)$.
From Fig. 3b and (7) we find that the threshold upcrossings of $v(t)$ come in clusters and they can only occur after $c(t)$ has an up-crossing event. Therefore, the first upward threshold-crossing of $v(t)$ occurs after the first up-crossing of $c(t)$. The actual signal crossing will have a uniformly distributed time delay in the range of $\left[0,1 / f_{c}\right]$ with respect to the envelope up-crossing. In this case, the PDF of the first-threshold-crossing probability of the envelop $c(t)$ can be considered as the first-thresholdcrossing probability of the band-pass noise $v(t)$, where the actual detection moment shows an extra delay of on average $\left[0,1 / 2 f_{c}\right]$. As a result, the rate of upward crossings $N_{T H}^{+}$in this case is

$$
N_{T H}^{+}=\left.\int_{0}^{\infty} \dot{c} p(c, \dot{c} ; t) d \dot{c}\right|_{c(t)=V_{T H}},
$$

where $c(t)$ and $\dot{c}(t)$ are independent, $\dot{c}(t)$ has a Gaussian distribution and $p(c, \dot{c} ; t)$ is the joint PDF for $c(t)$ and $\dot{c}(t)$.

In order to obtain all the parameters in the joint PDF of $p(v, \dot{v} ; t)$ and $p(c, \dot{c} ; t)$, we need to calculate the variance of $v(t), \dot{v}(t), c(t)$, and $\dot{c}(t)$, respectively. According to the Wiener-Khinchine relations,

$$
\begin{aligned}
& R_{v v}(\tau)=\int_{-\infty}^{\infty} \mathcal{P}_{v v}(f) e^{j 2 \pi f \tau} d f, \\
& R_{\dot{v} \dot{v}}(\tau)=\int_{-\infty}^{\infty} \mathcal{P}_{\dot{v} \dot{v}}(f) e^{j 2 \pi f \tau} d f,
\end{aligned}
$$

where $R_{v v}$ and $R_{\dot{v} \dot{v}}$ are the autocorrelation functions of the random processes $v(t)$ and $\dot{v}(t)$, respectively. $\mathcal{P}_{v v}(f)$ and $\mathcal{P}_{\dot{v} \dot{v}}(f)$ are the PSD of $v(t)$ and $\dot{v}(t)$, respectively, and they have the relation $\mathcal{P}_{\dot{v} \dot{v}}(f)=(2 \pi f)^{2} \mathcal{P}_{v v}(f)$. Therefore, we obtain the relation that

$$
\begin{aligned}
R_{v v}(\tau)^{\prime \prime} & =\int_{-\infty}^{\infty}(j 2 \pi f)^{2} \mathcal{P}_{v v}(f) e^{j 2 \pi f \tau} d f \\
& =-R_{\dot{v} \dot{v}}(\tau),
\end{aligned}
$$

where " indicates the second derivative operator.

Let $\psi_{0}=\left.R_{v v}(\tau)\right|_{\tau=0}$ and $\psi_{1}=\left.R_{\ddot{c} \dot{c}}(\tau)\right|_{\tau=0}$, which are the variances of $v(t)$ and $\dot{c}(t)$, respectively. Then, from (10), we have $\psi_{0}^{\prime \prime}=-\left.R_{\dot{v} \dot{v}}(\tau)\right|_{\tau=0}$, and from (7), we can get $\psi_{0}=$ $\left.R_{c c}(\tau)\right|_{\tau=0}$. Moreover, we determine $\psi_{1}$ by expanding the derivative of (7) as

$$
\begin{aligned}
\dot{v}(t)= & \dot{c}(t) \cos \left(2 \pi f_{c} t+\theta(t)\right)- \\
& c(t)\left(2 \pi f_{c}+\dot{\theta}(t)\right)\left[\sin \left(2 \pi f_{c} t+\theta(t)\right)\right],
\end{aligned}
$$

where (11) is a linear combination of two independent random processes, and its variance is the sum of the two variances and reduces to

$$
-\psi_{0}^{\prime \prime}=\psi_{1}+\left(2 \pi f_{c}\right)^{2} \psi_{0} .
$$


In the receiver of Fig. 1, the input signal as well as the noise is filtered by an ideal band-pass filter. For band-limit noise,

$$
\begin{aligned}
& \psi_{0} \triangleq \sigma_{B}^{2}=2 \int_{f_{L}}^{f_{H}} \mathcal{P}_{v v}(f) d f=2 \sigma_{o}^{2}\left(f_{H}-f_{L}\right) . \\
& \psi_{0}^{\prime \prime}=2 \int_{f_{L}}^{f_{H}}(j 2 \pi f)^{2} \mathcal{P}_{v v}(f) d f=-\frac{8}{3} \pi^{2} \sigma_{0}^{2}\left(f_{H}^{3}-f_{L}^{3}\right) .
\end{aligned}
$$

Let $\lambda_{0}=\frac{1}{\pi} \sqrt{-\frac{\psi_{0}^{\prime \prime}}{\psi_{0}}}=\sqrt{\frac{4}{3} \frac{f_{H}^{3}-f_{L}^{3}}{f_{H}-f_{L}}}$, then we have

$$
\psi_{1}=\lambda_{0}^{2} \pi^{2} \psi_{0} .
$$

Following the above derivation, $p(v, \dot{v} ; t)$ in (6) and $p(c, \dot{c} ; t)$ in $(8)$ can be deduced as

$$
\begin{aligned}
& p(v, \dot{v} ; t)=\frac{\left(-\psi_{0} \psi_{0}^{\prime \prime}\right)^{-1 / 2}}{2 \pi} e^{-\frac{v^{2}}{2 \psi_{0}}+\frac{\dot{v}^{2}}{2 \psi_{0}^{\prime \prime}}}, \\
& p(c, \dot{c} ; t)=\frac{c \psi_{0}^{-1} \psi_{1}^{-1 / 2}}{\sqrt{2 \pi}} e^{-\frac{c^{2}}{2 \psi_{0}}-\frac{\dot{c}^{2}}{2 \psi_{1}}} .
\end{aligned}
$$

Let $\phi(x)=\frac{1}{\sqrt{2 \pi}} e^{\left(-\frac{x^{2}}{2}\right)}$, then we find for the up-crossing rate $N_{T H}^{+}$for large $B / f_{c}$

$$
\begin{aligned}
N_{T H}^{+} & =\left.\int_{0}^{\infty} \dot{v} p(\nu, \dot{v} ; t) d \dot{v}\right|_{\nu(t)=V_{T H}} \\
& =\left.\left(-\psi_{0} \psi_{0}^{\prime \prime}\right)^{-1 / 2} \phi\left(\frac{v}{\sqrt{\psi_{0}}}\right) \int_{0}^{\infty} \dot{v} \phi\left(\frac{\dot{v}}{\sqrt{-\psi_{0}^{\prime \prime}}}\right) d \dot{v}\right|_{\nu(t)=V_{T H}} \\
& =\frac{1}{2} \lambda_{0} e^{-\frac{v_{T H}^{2}}{2 \sigma_{B}^{2}}} .
\end{aligned}
$$

In the case of smaller $B / f_{c}$, we find for the up-crossing rate $N_{T H}^{+}$

$$
\begin{aligned}
N_{T H}^{+} & =\left.\int_{0}^{\infty} \dot{c} p(c, \dot{c} ; t) d \dot{c}\right|_{c(t)=V_{T H}} \\
& =\left.\sqrt{2 \pi} c \psi_{0}^{-1} \psi_{1}^{-1 / 2} \phi\left(\frac{c}{\sqrt{\psi_{0}}}\right) \int_{0}^{\infty} \dot{c} \phi\left(\frac{\dot{c}}{\sqrt{\psi_{1}}}\right) d \dot{c}\right|_{c(t)=V_{T H}} \\
& =\sqrt{\frac{\pi}{6}} \frac{V_{T H}}{\sigma_{B}}\left(f_{H}-f_{L}\right) e^{-\frac{V_{T H}^{2}}{2 \sigma_{B}^{2}}} .
\end{aligned}
$$

Especially when

$$
\overline{f_{c}}=\frac{1}{\sqrt{\frac{\pi}{6}\left(\frac{V_{T H}}{\sigma_{B}}\right)^{2}-\frac{1}{12}}} .
$$

(17) and (18) are equal. We can use (19) as the division of relatively "narrow-band" case and "wide-band" case.
Now (5), (17), and (18) reveal that $N_{T H}^{+}$and the PDF of the first-crossing time $p_{c}\left(V_{T H}, t\right)$ can be computed from the pre-set parameters $V_{T H}, f_{H}, f_{L}$, and the PSD of the noise $\sigma_{0}^{2}$. Moreover, the PDF of a single TOA measurement and the joint PDF of a number of TOA measurements can be obtained based on $p_{c}\left(V_{T H}, t\right)$, which will be further discussed in Sections 3.2 and 3.3. However, when $\sigma_{0}$ is unknown, it can be estimated from the empirical PDF of the first-crossing time which can be obtained from a set of TOA measurements, as will be shown in Sections 3.2 and 3.3 .

\subsection{PDF of a single TOA measurement}

Consider the PDF of the $k$-th pulse arrival time measurement, recorded as $t_{M}[k]$. In the noise-only case, as indicated in (5), this PDF is

$$
p\left(t_{M}[k] ; \mu_{1}\right)=\mu_{1} e^{-\mu_{1} t_{M}[k]} \quad t_{M}[k]>0,
$$

where $\mu_{1}=N_{T H}^{+}$is a constant which can be calculated from (17) or (18) when $\sigma_{0}$ is known, or it can be estimated by (24) when $\sigma_{0}$ is unknown.

In case a pulse is received, the PDF of $t_{M}[k]$ becomes

$p\left(t_{M}[k] ; t_{r}, \mu_{1}, \mu_{2}\right)= \begin{cases}p_{e}\left(t_{M}[k] ; \mu_{1}\right) & 0<t_{M}[k]<t_{r} \\ p_{d}\left(t_{M}[k] ; t_{r}, \mu_{1}, \mu_{2}\right) & t_{r} \leq t_{M}[k] \leq t_{f} \\ p_{m}\left(t_{M}[k] ; t_{r}, \mu_{1}, \mu_{2}\right) & t_{M}[k]>t_{f}\end{cases}$

where

$$
\begin{aligned}
p_{e}\left(t_{M}[k] ; \mu_{1}\right) & =\mu_{1} e^{-\mu_{1} t_{M}[k]}, \\
p_{d}\left(t_{M}[k] ; t_{r}, \mu_{1}, \mu_{2}\right) & =\mu_{2} e^{-\left(\mu_{1}-\mu_{2}\right) t_{r}} e^{-\mu_{2} t_{M}[k]}, \\
p_{m}\left(t_{M}[k] ; t_{r}, \mu_{1}, \mu_{2}\right) & =\mu_{1} e^{\left(\mu_{1}-\mu_{2}\right)\left(t_{f}-t_{r}\right)} e^{-\mu_{1} t_{M}[k]} .
\end{aligned}
$$

and $\mu_{2}$ is the coefficient of the PDF for the pulse duration where $V_{T H}=\left(V_{N}-A\right)$ as shown in Fig. 2e. The value of $\mu_{2}$ can be calculated from (17) or (18) when it satisfies the "rare crossing" criterion proposed in [24] $\left(V_{T H}>2 \sigma_{B}\right.$ for a wide-band process); otherwise, it can be estimated by (24). When $\mu_{2}=\mu_{1}$, (21) becomes (20); thus, the PDF of $t_{M}[k]$ can be unified as (21), of which (20) is a special case. Figure 4 shows an example of the PDF of a single FP pulse arrival time measurement in the case of (20) and (21), respectively, which were fitted to the histogram of TOA measurements. Figure $4 \mathrm{a}$ is obtained under the condition of $f_{L}=3.1 \mathrm{GHz}, f_{H}=6.1 \mathrm{GHz}, V_{N} / \sigma_{B}=1.7$, where the horizontal axes indicates the measured TOA, and Fig.4b is obtained when the FP arrives at $t_{r}=2 \mathrm{~ns}$ with a firstpeak-to-noise-ratio (FPNR) of FPNR $=0.5 \mathrm{~dB}$ based on the noise floor in Fig. 4a, where FPNR $=10 \log \left(A^{2} / \sigma_{B}^{2}\right)$.

Moreover, we can see from (21) that, it is a segmented PDF. Based on this PDF, we define the probability of 


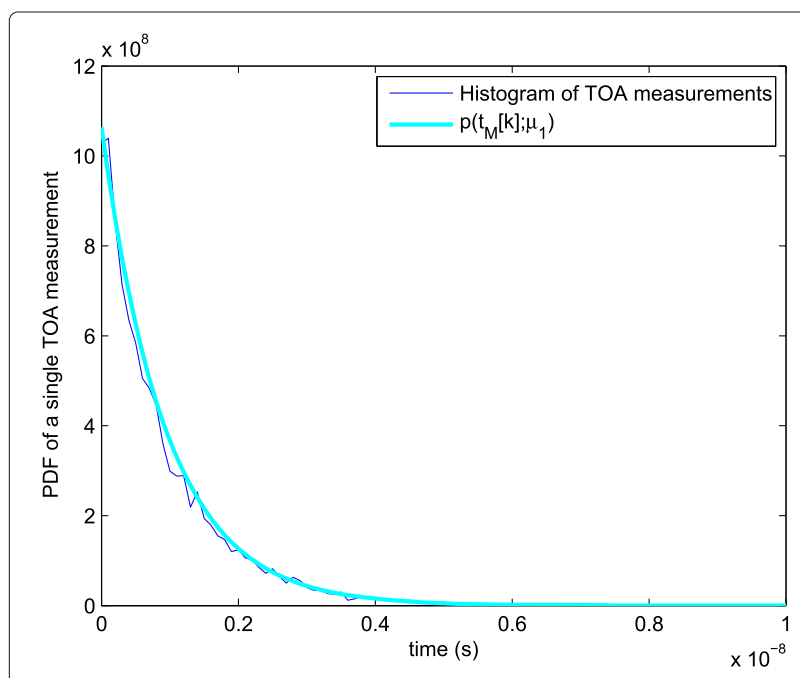

(a) PDF for the noise-only case

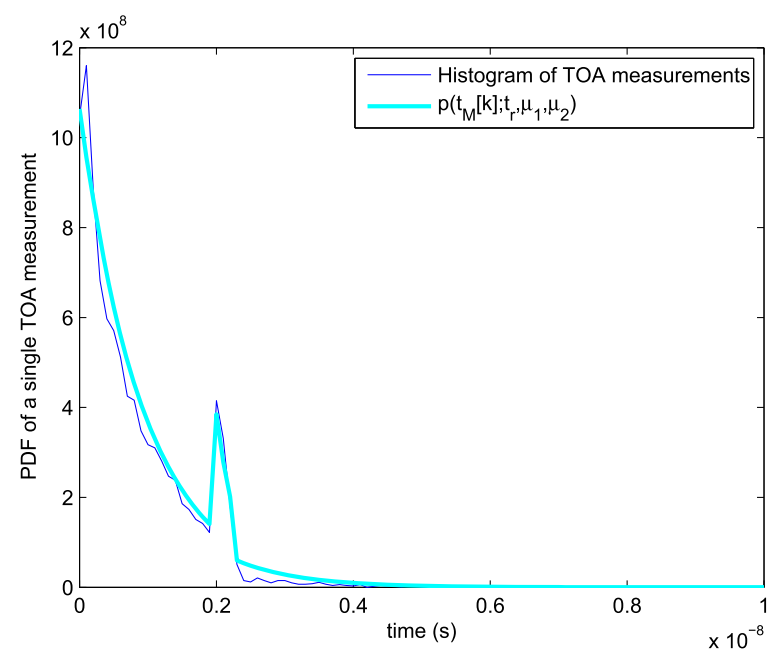

(b) PDF for the case with a pulse received

Fig. 4 PDF of the threshold-crossing moment for a the noise-only case, $\mathbf{b}$ the case with a single received FP pulse at $t_{r}=2 \mathrm{~ns}$ and $F P N R=0.5 \mathrm{~dB}$. The bandpass noise signal is white within $f_{L}=3.1 \mathrm{GHz}$ and $f_{H}=6.1 \mathrm{GHz}$ and $V_{N} / \sigma_{B}=1.7$

early false alarm $\left(P_{E F A}\right)$, probability of detection $\left(P_{D}\right)$, and probability of missed detection $\left(P_{M D}\right)$ as

$P_{E F A} \triangleq$ probability of a TOA measurement which occurs in the time interval before the FP pulse arrives\},

$P_{D} \triangleq$ probability of a TOA measurement which occurs in the time interval of the FP pulse\},

$P_{M D} \triangleq$ \{probability of a TOA measurement which occurs in the time interval after the FP pulse\}.
Accordingly, we can get the equations for these probabilities as

$$
\begin{aligned}
P_{E F A} & =\int_{0}^{t_{r}} p_{e}\left(t_{M}[k] ; \mu_{1}\right) d t_{M}[k]=1-e^{-\mu_{1} t_{r}}, \\
P_{D} & =\int_{t_{r}}^{t_{f}} p_{d}\left(t_{M}[k] ; t_{r}, \mu_{1}, \mu_{2}\right) d t_{M}[k] \\
& =e^{-\mu_{1} t_{r}}\left(1-e^{-\mu_{2} t_{w}}\right), \\
P_{M D} & =\int_{t_{f}}^{\infty} p_{m}\left(t_{M}[k] ; t_{r}, \mu_{1}, \mu_{2}\right) d t_{M}[k] \\
& =e^{-\mu_{1} t_{r}} e^{-\mu_{2} t_{w}},
\end{aligned}
$$

where $t_{w}=t_{f}-t_{r}$.

Moreover, $P_{E F A}$ and $P_{M D}$ can both be categorized as $P_{F A}$; thus, we have $P_{F A}=P_{E F A}+P_{M D}$.

Based on (22), Fig. 5 gives an overview on how the variables $t_{r}, V_{N} / \sigma_{B}$, and $F P N R$ affect $P_{E F A}, P_{D}$ and $P_{M D}$. Specifically, Fig. 5 indicates that the interval between the start of the measurement and the arrival time of the pulse $t_{r}$ has a significant influence on $P_{E F A}, P_{D}$, and $P_{M D}$ besides of the obvious impact of $V_{N} / \sigma_{B}$ and $F P N R$ as discussed in [1] and [2]. Figure 5a and (22) show that $V_{N} / \sigma_{B}$ and $t_{r}$ are factors which would influence $P_{E F A} . P_{E F A}$ is the probability of triggering by noise which increases with $t_{r}$. Moreover, $\mu_{1}$ in (22), which depends on $V_{N} / \sigma_{B}$ according to (17) and (20), determines $P_{E F A}$, and a lower $V_{N} / \sigma_{B}$ results in larger $P_{E F A}$ because at a lower $V_{N}$, level-crossing by noise becomes more likely. Figure $5 \mathrm{~b}$ indicates that $F P N R, V_{N} / \sigma_{B}$, and $t_{r}$ influence $P_{D}$. For a fixed $F P N R$, a lower $V_{N} / \sigma_{B}$ results in a larger negative slope in $P_{D}$ which increases with $t_{r}$ because of the increased $P_{E F A}$, and larger $F P N R$ leads to a higher $P_{D}$ for a fixed $V_{N} / \sigma_{B}$. This can be explained as, when $V_{N}$ is lower, the input signal has a larger probability to cross $V_{N}$, while when $t_{r}$ is larger, $P_{E F A}$ increases due to the increased probability of triggering due to noise before the pulse has arrived. For the same $V_{N} / \sigma_{B}$, a larger $F P N R$ will increase $P_{D}$, since for a larger amplitude of the FP pulse it will be more likely to be detected. Similarly, Fig. $5 \mathrm{c}$ shows the relation between FPNR, $V_{N} / \sigma_{B}, t_{r}$, and $P_{M D}$ that FPNR and $V_{N} / \sigma_{B}$ influence the initial offset of $P_{M D}$ and $V_{N} / \sigma_{B}$ has a major impact on the slope of $P_{M D}$.

\subsection{Composite hypothesis testing}

Since the distribution of the measured level-crossing times in the noise-only case $(F P N R=0)$ is different from that in case a pulse is present $(F P N R \neq 0)$, as shown in Fig. 4, this characteristic can be exploited in detection of the pulse from the noise floor using composite hypothesis testing.

\subsubsection{The GLRT detector}

Let us represent $K$-independent TOA measurements as the vector $\boldsymbol{t}_{M}$, which are sorted in an ascending order: 


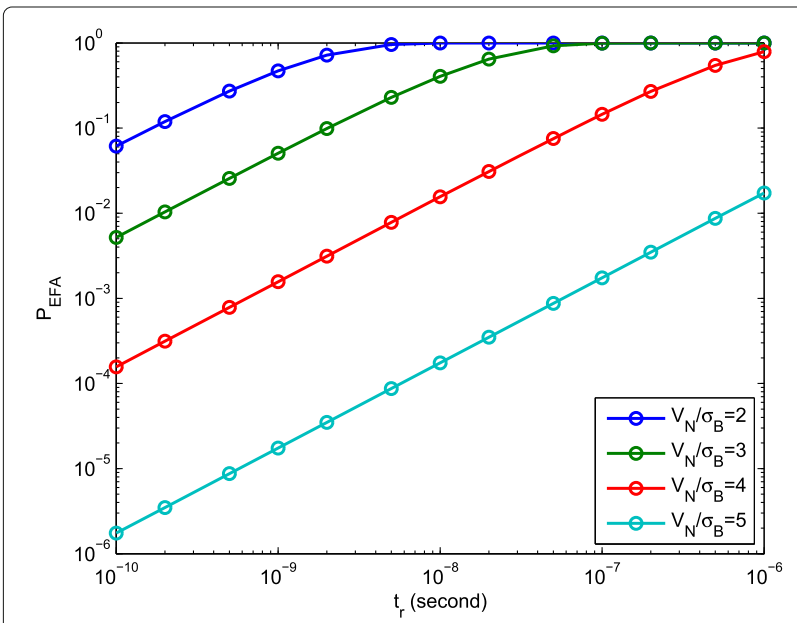

(a) $P_{E F A}$ verses $t_{r}$ under different $V_{N} / \sigma_{B}$

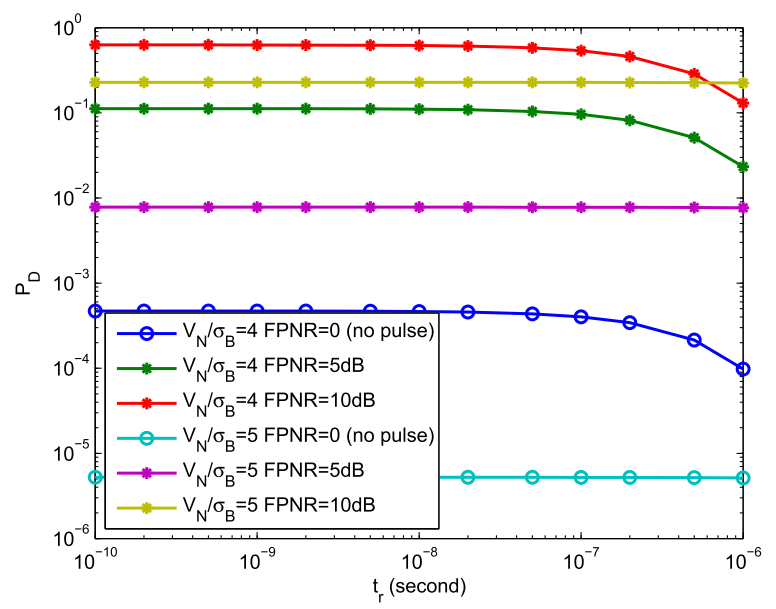

(b) $P_{D}$ verses $t_{r}$ under different $V_{N} / \sigma_{B}$ and $F P N R$

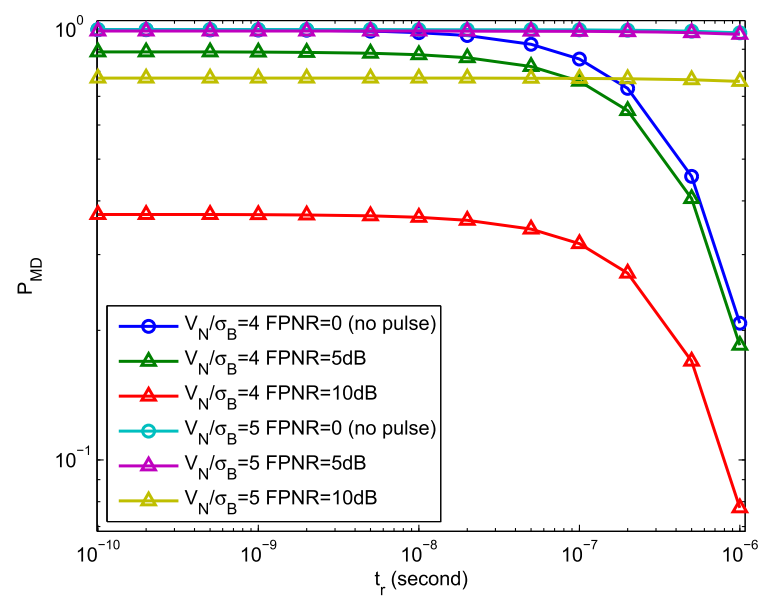

(c) $P_{M D}$ verses $t_{r}$ under different $V_{N} / \sigma_{B}$ and $F P N R$

Fig. 5 Relation between $P_{E F A}, P_{D}$ and $P_{M D}$ as a function of the true FP arrival time $t_{r}$, and the parameters $V_{N} / \sigma_{B}$ and FPNR as deduced from (22)
$t_{M}[1] \leq \cdots t_{M}[k] \leq \cdots \leq t_{M}[K](k=1, \cdots, K)$, where $p$ and $q$ are the number of measurements which are located in the time interval $0<t_{M}[k]<t_{r}$ and $t_{r} \leq t_{M}[k] \leq$ $t_{f}$, respectively, i.e., $p+q \leq K$. Now, we consider the following two hypotheses

$\mathcal{H}_{0}: \mu_{1}=\mu_{2}, \quad$ noise-only case $(A=0)$,

$\mathcal{H}_{1}: \mu_{1} \neq \mu_{2}, \quad$ pulse received case $(A>0$ or $A<0)$.

A GLRT detector decides $\mathcal{H}_{1}$ if

$$
\begin{aligned}
L\left(\boldsymbol{t}_{M}\right) & =\frac{p\left(\boldsymbol{t}_{M} ; \hat{t}_{r}, \hat{\mu}_{1}, \hat{\mu}_{2}, \mathcal{H}_{1}\right)}{p\left(\boldsymbol{t}_{M} ; \hat{\mu}_{1}, \mathcal{H}_{0}\right)} \\
& =\max _{t_{r}, \mu_{1}, \mu_{2}} \frac{p\left(\boldsymbol{t}_{M} ; t_{r}, \mu_{1}, \mu_{2}, \mathcal{H}_{1}\right)}{p\left(\boldsymbol{t}_{M} ; \mu_{1}, \mathcal{H}_{0}\right)}>\gamma,
\end{aligned}
$$

where $\hat{t}_{r}$ is the MLE of the TOA under $\mathcal{H}_{1}, \gamma$ is the threshold of the GLRT detector and $p\left(\boldsymbol{t}_{M}\right)$ is the joint PDF of (21), which is

$$
\begin{aligned}
p\left(\boldsymbol{t}_{M} ; \mu_{1}, \mathcal{H}_{0}\right) & =\prod_{k=1}^{K} p_{e}\left(t_{M}[k] ; \mu_{1}\right), \\
p\left(\boldsymbol{t}_{M} ; t_{r}, \mu_{1}, \mu_{2}, \mathcal{H}_{1}\right)= & \prod_{k=1}^{p} p_{e}\left(t_{M}[k] ; \mu_{1}\right) . \\
& \prod_{k=p+1}^{p+q} p_{d}\left(t_{M}[k] ; t_{r}, \mu_{1}, \mu_{2}\right) . \\
& \prod_{k=p+q+1}^{K} p_{m}\left(t_{M}[k] ; t_{r}, \mu_{1}, \mu_{2}\right),
\end{aligned}
$$

where '.' is the multiplication operator.

Furthermore, when $\sigma_{0}^{2}$ is unknown, $\hat{\mu}_{1}$ and $\hat{\mu}_{2}$ can be obtained by fitting $P_{E F A}$ and $P_{D}$ to the empirical statistics of the TOA measurements as follows

$$
\begin{aligned}
P_{E F A} & =\int_{0}^{t_{r}} p_{e}\left(t_{M}[k] ; \hat{\mu}_{1}\right) d t_{M}[k]=\frac{p}{K}, \\
P_{D} & =\int_{t_{r}}^{t_{f}} p_{d}\left(t_{M}[k] ; t_{r}, \hat{\mu}_{1}, \hat{\mu}_{2}\right) d t_{M}[k]=\frac{q}{K} .
\end{aligned}
$$

The final estimated TOA $\hat{t}_{r}$ is found by maximizing (23) over all possible values of $t_{r}$ [25]. The search step of $t_{r}$ can be chosen according to the resolution of $t_{M}[k]$. To be specific, during every search step of $t_{r}, p$, and $q$ are first calculated and then $\hat{\mu}_{1}$ and $\hat{\mu}_{2}$ are obtained from (24).

\subsubsection{The optimal GLRT detector}

In (23), the threshold of the detector $\gamma$ is to be determined. In order to optimize the setting of $\gamma$, we use the idea of the Neyman-Pearson lemma, which is based on a single observation, for multiple observations. And choose $\gamma$ by setting the probability of false alarm $P_{F A}$ equal to $\alpha$, as

$$
P_{F A}=\int_{\left\{\boldsymbol{t}_{M}: L\left(\boldsymbol{t}_{M}\right)>\gamma\right\}} p\left(\boldsymbol{t}_{M} ; \hat{\mu}_{1}, \mathcal{H}_{0}\right) d \boldsymbol{t}_{M}=\alpha .
$$


Let $u(k)=t_{M}[k]-\hat{t}_{r},(k=p+1, \cdots, p+q)$, where $u(k)$ conforms to a truncated exponential distribution $u(k) \sim \operatorname{Exp}\left(\hat{\mu}_{1}\right), \quad u(k) \in\left(0, t_{w}\right)$. Set the test statistic $T \triangleq$ $\sum_{k=p+1}^{p+q} u(k)$. The critical region $\left\{\boldsymbol{t}_{M}: L\left(\boldsymbol{t}_{M}\right)>\gamma\right\}$ in (25) can be deduced to $T \lessgtr \gamma^{\prime}$ (see Appendix). Accordingly, (25) can be re-written as

$$
P_{F A}=\int_{\left\{T \lessgtr \gamma^{\prime}\right\}} p\left(T ; \hat{\mu}_{1}, \mathcal{H}_{0}\right) d T=\alpha .
$$

Bain and Weeks [26] indicates that the PDF of $p\left(T ; \hat{\mu}_{1}, \mathcal{H}_{0}\right)$ is given by

$$
\begin{aligned}
& p\left(T ; \hat{\mu}_{1}, \mathcal{H}_{0}\right) \\
& =\frac{\left(\frac{\hat{\mu}_{1}}{1-e^{-\hat{\mu}_{1} t_{w}}}\right)^{q}}{(q-1) !} e^{-\hat{\mu}_{1} T} \sum_{\beta=0}^{\beta_{0}}(-1)^{\beta}\left(\begin{array}{c}
q \\
\beta
\end{array}\right)\left(T-\beta t_{w}\right)^{q-1} \\
& \left(\beta_{0} t_{w}<T<\left(\beta_{0}+1\right) t_{w}\right),
\end{aligned}
$$

where $\beta$ and $\beta_{0}$ are integers and $\beta_{0}$ belongs to the set of $\beta_{0} \in[0,1, \cdots,(q-1)]$.

\subsubsection{Joint PDF approximation approach}

Since the calculation of (27) has the potential risk of overflowing machine precision when $q$ is large, an approximation of (27) can be used as a sub-optimal solution in practice.

Monte Carlo simulation based on the empirical histogram of $T$ shows that $p\left(T ; \hat{\mu}_{1}, \mathcal{H}_{0}\right)$ can be fitted to the Erlang distribution $T \sim \mathcal{E} \operatorname{rlang}(A p, B t)$ or the Normal distribution $T \sim \mathcal{N}$ ormal $(M u$, Sig), where $A p$ and $B t$ are the shape and the rate of the Erlang distribution, respectively, and $\mathrm{Mu}$ and Sig are the mean and standard deviation of the Normal distribution, respectively. These parameters can be calibrated from

$$
\begin{aligned}
A p & =\frac{\left(\mathrm{E}\left(\sum_{k=p+1}^{p+q} u(k)\right)\right)^{2}}{\operatorname{var}\left(\sum_{k=p+1}^{p+q} u(k)\right)}=\frac{\left(\sum_{k=p+1}^{p+q} \mathrm{E}(u(k))\right)^{2}}{\sum_{k=p+1}^{p+q} \operatorname{var}(u(k))}, \\
B t & =\frac{\mathrm{E}\left(\sum_{k=p+1}^{p+q} u(k)\right)}{\operatorname{var}\left(\sum_{k=p+1}^{p+q} u(k)\right)}=\frac{\sum_{k=p+1}^{p+q} \mathrm{E}(u(k))}{\sum_{k=p+1}^{p+q} \operatorname{var}(u(k))}, \\
M u & =q \mathrm{E}(u(k)), \\
\text { Sig } & =\sqrt{q \operatorname{var}(u(k))} .
\end{aligned}
$$

Here, $\mathrm{E}(u(k))$ and $\operatorname{var}(u(k))$ are the mean and the variance of $u(k)$, respectively, which can be obtained by

$$
\begin{aligned}
\mathrm{E}(u(k)) & =\int_{0}^{t_{w}} u(k) f(u(k)) d u(k)=\frac{1}{\hat{\mu}_{1}}-\frac{t_{w} e^{-\hat{\mu}_{1} t_{w}}}{1-e^{-\hat{\mu}_{1} t_{w}}}, \\
\operatorname{var}(u(k)) & =E\left(u(k)^{2}\right)-(E(u(k)))^{2} \\
& =\int_{0}^{t_{w}} u(k)^{2} f(u(k)) d u(k)-\int_{0}^{t_{w}} u(k) f(u(k)) d u(k) \\
& =\frac{1}{\hat{\mu}_{1}^{2}}-\frac{t_{w}^{2} e^{-\hat{\mu}_{1} t_{w}}}{\left(1-e^{-\hat{\mu}_{1} t_{w}}\right)^{2}},
\end{aligned}
$$

where $f(u(k))=\hat{\mu}_{1} e^{-\hat{\mu}_{1} u(k)}$.

Under these approximations, the goodness of fit of the Erlang approximation and the Normal approximation were tested using Monte Carlo integration [27]. According to (26), the pre-set $P_{F A}=\alpha$ leads to a corresponding threshold $\gamma^{\prime}$ by means of calculating the inverse cumulative density function (CDF) of $T$. However, a $\gamma^{\prime}$ could be biased due to the approximation of $p\left(T ; \hat{\mu}_{1}, \mathcal{H}_{0}\right)$, which would lead to a biased $P_{F A}$ accordingly, compared to the usage of the true $p\left(T ; \hat{\mu}_{1}, \mathcal{H}_{0}\right)$. Since (27) is only obtainable when $q$ is small, we take $P_{F A}$ obtained by Monte Carlo integration as a reference to evaluate these two possible approximations. Figure 6 shows the results of the bias in $P_{F A}$ for $V_{N} / \sigma_{B}=2$ (low $V_{N} / \sigma_{B}$ case) and $V_{N} / \sigma_{B}=4$ (desired $V_{N} / \sigma_{B}$ case), where the bias in $P_{F A}$ under the two approximations are compared to the $P_{F A}$ obtained from the Monte Carlo integration. It is seen that, when $q$ is large enough $(q>10)$, it is feasible to use either the Normal or the Erlang distribution to approximate $p\left(T ; \hat{\mu}_{1}, \mathcal{H}_{0}\right)$ in (27) with less than $1 \%$ bias in $P_{F A}$ under the given conditions.

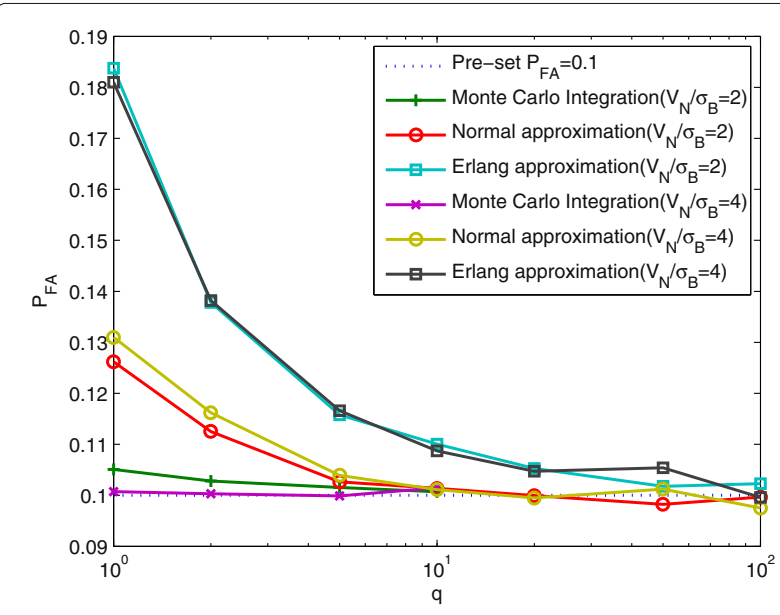

Fig. 6 Comparison of the joint PDF approximation tests showing the bias in $P_{F A}$ due to the joint PDF approximation (Erlang approximation and Normal approximation to (27)) applied to (26), when the empirical histogram obtained from Monte Caro integration is taken as reference 


\section{Simulation results}

In the simulations, we use the following parameters: a BPF with $f_{L}=3.1 \mathrm{GHz}$ and $f_{H}=6.1 \mathrm{GHz}$, i.e., $f_{c}=4.6 \mathrm{GHz}$ and $B=3 \mathrm{GHz}$, the number of measured arrival time events $K=100, P_{F A}=0.1$, and the resolution of each recorded TOA measurement is assumed to be $10 \mathrm{ps}$. The TOA estimation results are shown in Figs. 7, 8, and 9, where the horizontal axis indicates the true TOA $t_{r}$ and the vertical axis gives the mean absolute estimation error.

In [2], the measurement window is devided in a number of time bins. It is shown that the TOA $t_{r}$ is within the first time bin of the measurement window which contains at least $K_{T H}$ out of $K$ measured TOA's, where $1 \leq K_{T H} \leq K$. Specifically, a 160 ns time window at the start of each measurement window is excluded from the analysis because of the high probability of triggering due to noise. The ratio $V_{N} / \sigma_{B} \in[3.5,5.5]$ is selected according to the chosen bounds on $P_{E F A}$ and $P_{M D}$.

The results of Fig.7 show that under the conditions where $V_{N} / \sigma_{B}$ and $F P N R$ are set relatively high, both the proposed GLRT detector-based TOA estimation and the time-window-based TOA estimation from [2], realize centimeter-level ranging accuracy. The TOA estimation scheme proposed in this paper shows improved performance compared to the scheme used in [2].

Compared to [2], the GLRT-based pulse detection and TOA estimation scheme works in the whole measurements window, without any unreachable time range as shown in Fig. 7, for this scheme detects the pulse as well as estimates its arrival time based on the ratio of the PDFs of the pulse-present case and noise-only case. Since in [2] the start of the measurement period is discarded from the

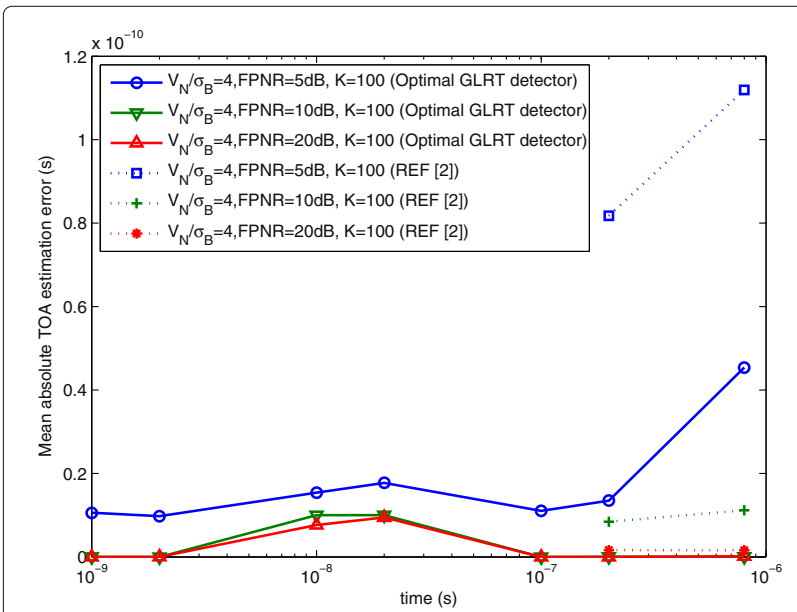

Fig. $7 \mathrm{TOA}$ estimation error for $V_{N} / \sigma_{B}=4$ with FPNR as parameter. This figure compares the TOA estimation error between the proposed GLRT detector and the time-window-based detector of [2] with the true TOA $t_{r}$ ranging from $1 \mathrm{~ns}$ to $1 \mu \mathrm{s}$, where the time-window-based detector only works in the $t_{r}$ range from 160 ns to $1 \mu \mathrm{s}$

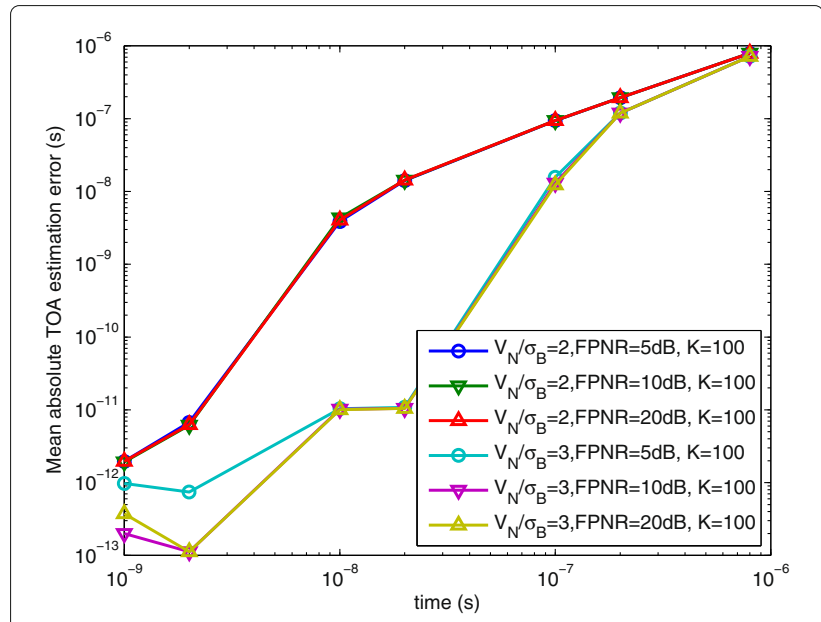

Fig. 8 TOA estimation error for low $V_{N} / \sigma_{B}$ with FPNR as parameter

measurements, a pulse cannot be detected when it arrives within this $160 \mathrm{~ns}$ window. The improved performance of the proposed technique is because it is an MLE scheme based on the PDFs of the measured data, rather than a time-window-oriented detection scheme, which better exploits the available information of the measurement data. But this comes at the expense of a higher computational cost. Moreover, the proposed scheme could work under a wider range of $V_{N} / \sigma_{B}$ and $F P N R$ under specific conditions (e.g., specific range of $t_{r}$ ), as shown in Figs. 8 and 9, which is not applicable for the scheme in [2].

Figure 8 shows the performance of the proposed scheme for lower values of $V_{N} / \sigma_{B}$. For the cases of $V_{N} / \sigma_{B}=$ 2 and 3 , it is observed that the TOA estimation error increases with increasing $t_{r}$. This is due to the fact that $P_{E F A}$ increases and and $P_{D}$ decreases with increasing $t_{r}$ as shown in Fig. 5a, b, respectively. Also, $P_{D}$ decreases faster for smaller $V_{N} / \sigma_{B}$.

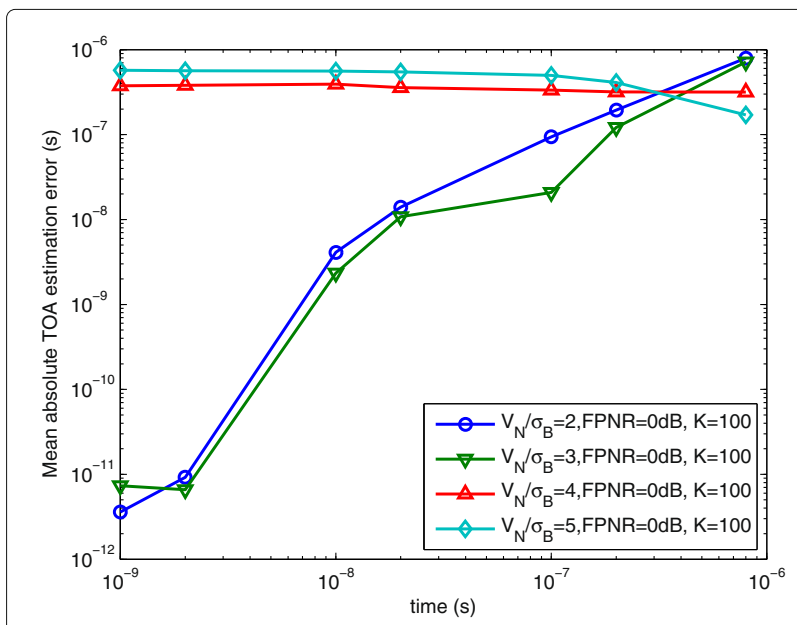

Fig. 9 TOA estimation error for low FPNR with $V_{N} / \sigma_{B}$ as parameter 
Figure 9 shows the TOA estimation accuracy when receiving a weak pulse signal. In case of high $V_{N} / \sigma_{B}$ and low FPNR where $V_{N} / \sigma_{B}=4$ and 5 and $F P N R=0 \mathrm{~dB}$, the threshold $V_{T H}$ shown in (3) is relatively high compared to $\sigma_{B}$, resulting in a low probability of detection of the FP pulse, but instead triggering of the detector happens mainly due to noise, i.e., resulting in EFAs and MDs. For low $V_{N} / \sigma_{B}$ and low FPNR, e.g., with $V_{N} / \sigma_{B}=2$ and 3 and $F P N R=0 \mathrm{~dB}$, it is possible to still detect the FP pulse, even when $t_{r}$ is relatively small, because it has a larger probability to cross the lower $V_{N}$. However, due to the low $V_{N} / \sigma_{B}$, a large fraction of the threshold crossings will be due to noise and cause EFAs when $t_{r}$ increases; therefore, the TOA estimation error is higher.

By comparing Figs. 7, 8, and 9, we find the trade-off between the sensitivity of the receiver and the accuracy of TOA estimation. When setting $V_{N} / \sigma_{B}$ high, a more accurate estimation can be obtained for high FPNR, as shown in Fig. 7. However, a high $V_{N} / \sigma_{B}$ also causes the missing of weak FP signals, as shown in Fig. 9. On the other hand, a low $V_{N} / \sigma_{B}$ setting will increase the sensitivity of the receiver but it worsens the estimation accuracy, especially when $t_{r}$ is large, as shown in Figs. 8 and 9. In general, the above analysis provides an approach to an optimal trade-off by selecting relatively low $V_{N} / \sigma_{B}$ according to the expected range of $t_{r}$, so as to realize centimeter-level TOA estimation accuracy.

In Fig. 10, the effect of the number $K$ of TOA measurements on the TOA estimation accuracy is shown while keeping $V_{N} / \sigma_{B}$ and FPNR constant. It is observed that the TOA estimation accuracy improves with increasing $K$. This is because the unknown parameters $\mu_{1}$ and $\mu_{2}$ in the GLRT detector are estimated from the empirical histogram deduced from the number of TOA measurements by (24). Therefore, the estimation accuracy of $\mu_{1}$ and $\mu_{2}$ as

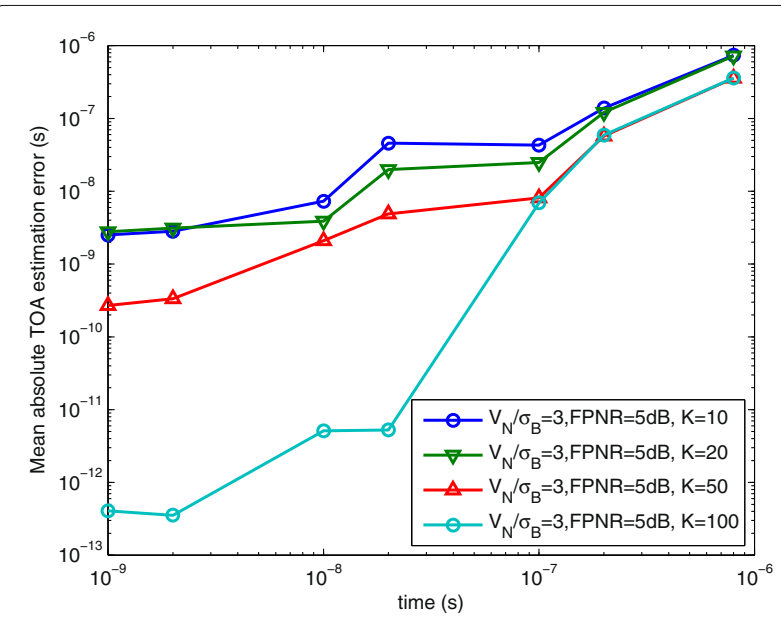

Fig. 10 TOA estimation error for different numbers of TOA measurements $K$ well as $t_{r}$ are improved when the number of the collected samples is larger.

\section{Conclusions}

An improved TOA estimation scheme for UWB pulse signals is proposed for a sub-Nyquist sampling receiver based on first-threshold-level crossing using statistical analysis of a number of measured arrival time events. The threshold crossing procedure of the receiver for the noise-only case and the case when a pulse plus noise is received has been unified to a common first-crossing problem in a continuous random process. Based on this mathematical model, the PDF of the arrival time events was deduced under two categories of receiver bandwidth. An analysis is given on how to estimate the PSD of the noise $\sigma_{0}^{2}$ based on empirical measurement data and how the parameters threshold to band-limited noise deviation-ratio $V_{N} / \sigma_{B}$, first-peak-to-noise-ratio FPNR, and true TOA $t_{r}$ affect the probability of early false alarm $P_{E F A}$, probability of detection $P_{D}$, and probability of missed detection $P_{M D}$. For estimation of the TOA from a number of measured arrival time events, a composite hypothesis test and MLE is applied. Compared to the TOA estimation strategy used in [2], this pulse detection and TOA estimation approach make use of the PDF of TOA measurements data instead of statistical data on probability; therefore, more information is obtained and exploited, resulting in a more accurate TOA estimation within a wider time frame range.

\section{Appendix}

Proof of the critical region transformation in NP lemma strategy

The critical region in (25) is

$$
\begin{aligned}
\ln L\left(t_{M}\right) & =\ln \frac{p\left(\boldsymbol{t}_{M} ; \hat{t}_{r}, \hat{\mu}_{1}, \hat{\mu}_{2}, \mathcal{H}_{1}\right)}{p\left(\boldsymbol{t}_{M} ; \hat{\mu}_{1}, \mathcal{H}_{0}\right)} \\
& =q \ln \frac{\hat{\mu}_{2}}{\hat{\mu}_{1}}+\left(\hat{\mu}_{1}-\hat{\mu}_{2}\right)\left(\sum_{k=p+1}^{p+q} t_{M}[k]-q \hat{t}_{r}\right) \\
& +(K-p-q)\left(\hat{\mu}_{1}-\hat{\mu}_{2}\right) t_{w} \\
& \triangleq q \ln \frac{\hat{\mu}_{2}}{\hat{\mu}_{1}}+\left(\hat{\mu}_{1}-\hat{\mu}_{2}\right) T+(K-p-q)\left(\hat{\mu}_{1}-\hat{\mu}_{2}\right) t_{w} \\
& >\ln \gamma .
\end{aligned}
$$

Let $\gamma^{\prime} \triangleq \frac{\ln \gamma-q \ln \frac{\hat{\mu}_{2}}{\hat{\mu}_{1}}-(K-p-q)\left(\hat{\mu}_{1}-\hat{\mu}_{2}\right) t_{w}}{\hat{\mu}_{1}-\hat{\mu}_{2}}$, (29) can be deduced to

$$
\left\{\begin{array}{l}
T<\gamma^{\prime}, \text { when } \hat{\mu}_{1}<\hat{\mu}_{2}(A>0) \\
T>\gamma^{\prime}, \text { when } \hat{\mu}_{1}>\hat{\mu}_{2}(A<0)
\end{array}\right. \text {. }
$$




\section{Acknowledgements}

This research work is founded by the NWO HERE-2 project (No. 11951).

\section{Authors' contributions}

$Y X$ made the main contributions to the theorems, implementations, and drafting this paper. GJ is the advisor who directed this work and contributed to the theorems and revisions. SS and CT contributed to the GLRT detector part and revisions. All authors read and approved the final manuscript.

\section{Competing interests}

The authors declare that they have no competing interests.

\section{Publisher's Note}

Springer Nature remains neutral with regard to jurisdictional claims in published maps and institutional affiliations.

\section{Author details}

${ }^{1}$ Faculty of Electrical Engineering, Mathematics and Computer Science, Delft University of Technology, Mekelweg 4, 2628 CD Delft, The Netherlands.

2Faculty of Civil Engineering and Geosciences, Delft University of Technology, Stevinweg 1,2628 CN Delft, The Netherlands.

Received: 15 May 2017 Accepted: 31 August 2017

Published online: 19 September 2017

\section{References}

1. I Guvenc, Z Sahinoglu, Threshold selection for UWB TOA estimation based on kurtosis analysis. IEEE Commun. Lett. 9(12), 1025-1027 (2005)

2. G Bellusci, GJM Janssen, J Yan, CCJM Tiberius, Performance evaluation of a low-complexity receiver concept for TOA-based ultrawideband ranging. IEEE Trans. Veh. Technol. 61(9), 3825-3837 (2012). doi:10.1109/TVT.2012.2207749

3. J-Y Lee, RA Scholtz, Ranging in a dense multipath environment using an UWB radio link. IEEE J. Sel. Areas in Commun. 20(9), 1677-1683 (2002). doi:10.1109/JSAC.2002.805060

4. C Falsi, D Dardari, L Mucchi, MZ Win, Time of arrival estimation for UWB localizers in realistic environments. EURASIP J. Advances in Sig. Process. 2006(1), 032082 (2006)

5. V Lottici, A D'Andrea, U Mengali, Channel estimation for ultra-wideband communications. IEEE J. Sel. Areas in Commun. 20(9), 1638-1645 (2002)

6. H Boujemaa, M Siala, in Communications, 2001. ICC 2001. IEEE International Conference On. On a maximum likelihood delay acquisition algorithm, vol. 8 (IEEE, 2001), pp. 2510-2514

7. Z Sahinoglu, S Gezici, I Guvenc, Ultra-wideband positioning systems theoretical limits, ranging algorithms, and protocols. (Cambridge Univerisity Press, 2008). www.cambridge.org/9780521873093

8. SJ Howard, K Pahlavan, Measurement and analysis of the indoor radio channel in the frequency domain. IEEE Trans. Instrum. Meas. 39(5), 751-755 (1990)

9. K Pahlavan, P Krishnamurthy, A Beneat, Wideband radio propagation modeling for indoor geolocation applications. IEEE Commun. Mag. 36(4), 60-65 (1998)

10. SS Ghassemzadeh, R Jana, CW Rice, W Turin, V Tarokh, Measurement and modeling of an ultra-wide bandwidth indoor channel. IEEE Trans. Commun. 52(10), 1786-1796 (2004)

11. C-C Chong, SK Yong, A generic statistical-based UWB channel model for high-rise apartments. IEEE Trans. Antennas Propag. 53(8), 2389-2399 (2005)

12. Z Tarique, W Malik, D Edwards, Bandwidth requirements for accurate detection of direct path in multipath environment. Electron. Lett. 42(2), 100-102 (2006)

13. NA Alsindi, B Alavi, K Pahlavan, Measurement and modeling of ultrawideband TOA-based ranging in indoor multipath environments. IEEE Trans. Veh. Technol. 58(3), 1046-1058 (2009)

14. X Li, K Pahlavan, Super-resolution TOA estimation with diversity for indoor geolocation. IEEE Trans. Wirel. Commun. 3(1), 224-234 (2004)

15. H Saarnisaari, in Vehicular Technology Conference, 1997, IEEE 47th. TLS-ESPRIT in a time delay estimation, vol. 3 (IEEE, 1997), pp. 1619-1623

16. JL Paredes, GR Arce, Z Wang, Ultra-wideband compressed sensing: channel estimation. IEEE J. Sel. Top. Sig. Process. 1(3), 383-395 (2007). doi:10.1109/JSTSP.2007.906657
17. V Yajnanarayana, P Handel, Compressive sampling based UWB TOA estimator. arXiv preprint arXiv:1602.08615 (2016)

18. RJ Fontana, SJ Gunderson, in Ultra Wideband Systems and Technologies, 2002. Digest of Papers. 2002 IEEE Conference On. Ultra-wideband precision asset location system (IEEE, 2002), pp. 147-150

19. G Bellusci, GJM Janssen, J Yan, CCJM Tiberius, in 2009 IEEE International Conference on Ultra-Wideband. A low-complexity UWB receiver concept for TOA based indoor ranging, (2009), pp. 618-623. doi:10.1109/ICUWB.2009.5288687

20. G Bellusci, G Janssen, J Yan, C Tiberius, in Proceedings of the 22nd International Technical Meeting of The Satellite Division of the Institute of Navigation (ION GNSS 2009). A sub-sampling receiver architecture for ultra-wideband time of arrival based ranging, (2009), pp. 471-480

21. JR Rice, FP Beer, First-occurrence time of high-level crossings in a continuous random process. J. Acoust. Soc. Am. 39(2), 323-335 (1966). ASA. http://asa.scitation.org/doi/abs/10.1121/1.1909893

22. SO Rice, Mathematical Analysis of Random Noise. ([New York] : American Telephone and Telegraph Co, New York, 1944)

23. IS Bendat, AG Piersol, Random data: analysis and measurement procedures, Fourth Edition. (John Wiley \& Sons, Inc., New Jersey, 2012), pp. 109-171. doi:10.1002/9781118032428.ch5

24. SH Crandall, KL Chandiramani, RG Cook, Some first-passage problems in random vibration. J. Appl. Mech. 33, 532 (1966). doi:10.1115/1.3625118

25. SM Kay, Fundamentals of statistical signal processing: detection theory. Prentice Hall Signal Processing Series. (Prentice-Hall PTR, New Jersey, 1998)

26. LJ Bain, DL Weeks, A note on the truncated exponential distribution. Ann. Math. Statist. 35(3), 1366-1367 (1964). doi:10.1214/aoms/1177703298

27. PJG Teunissen, DG Simons, CCJM Tiberius, Probability and observation theory: an introduction. (Delft University of Technology, Delft, 2005)

\section{Submit your manuscript to a SpringerOpen ${ }^{\circ}$ journal and benefit from:}

- Convenient online submission

Rigorous peer review

- Open access: articles freely available online

- High visibility within the field

- Retaining the copyright to your article

Submit your next manuscript at $>$ springeropen.com 